\title{
Mapping Valence Electron Distribution of Iron-Based Superconductors using Quantitative CBED and Precession Electron Diffraction
}

\author{
Lijun $\mathrm{Wu}^{1}$, Chao $\mathrm{Ma}^{1,2}$, Binghui $\mathrm{Ge}^{1,2}$, Weiguo Yin ${ }^{1}$, and Yimei Zhu ${ }^{1}$ \\ 1. Condensed Matter Physics and Materials Science Department, Brookhaven National Laboratory, \\ Upton, NY 11973, USA. \\ 2. Beijing National Laboratory for Condensed Matter Physics, Institute of Physics, Chinese Academy of \\ Sciences, Beijing 100190, China.
}

The discovery of superconductivity with transition temperature $\left(T_{c}\right)$ up to $56 \mathrm{~K}$ in Fe-based superconductors (FeSCs) has provided a grand opportunity to explore high-temperature superconducting materials beyond copper containing oxides. Like in the cuprates, superconductivity in FeSCs emerges when the parent antiferromagnetic phase is suppressed, typically by introduction of dopant atoms, such as $\mathrm{Co}$ - or $\mathrm{Ni}$ - doped $\mathrm{BaFe}_{2} \mathrm{As} 2$. Unlike the oxygen anions sitting at the middle of the $\mathrm{Cu}-\mathrm{Cu}$ bonds in the cuprates, the anions in FeSCs are positioned above and below the Fe planes. This low-symmetry setup facilitates the anion polarization. The electronic oscillation of the anions has been shown to be a critical relevant degree of freedom in a one-orbital model for FeSCs. Whether this holds in real materials and how it interacts with the other active degree of freedom in the Fe planes are urgently needed to be elucidated. We address these important questions by probing the Co concentration dependence of the valence electron density distribution in $\mathrm{Ba}\left(\mathrm{Fe}_{1-\mathrm{x}} \mathrm{Co}_{\mathrm{x}}\right)_{2} \mathrm{As}_{2}$ (BFCA), a prototypical FeSC using quantitative CBED (Fig. 1(a-c)). With the combination of measured low-order structure factors (SFs) and density function theory (DFT) calculated high-order SFs, we retrieve the charge density distribution for undoped $\left(\mathrm{x}=0, T_{c}=0\right)$ and optimally doped BFCA $\left(\mathrm{x}=0.1, T_{c}=22.5 \mathrm{~K}\right)$ through multipole refinement. The resulting three-dimensional (3D) and two-dimensional (2D) difference electron density map (difference between experimentally measured electron density and superimposed isolated spherical atomic electron density) are shown in Fig.1(d-h). The Co concentration dependence is characterized by a significant increase in the out-of-plane component of the electronic dipole moment around the As anions and in the out-of-plane components of the quadruple moment around the Fe cations (Fig.1(i,j)), echoing the significant increase in $T_{c}$ and indicating a strong dipole-quadruple interaction between As and Fe atoms. The observations show a strong correlation among $T_{c}$, the electronic polarization of the anions, interorbital charge transfer on the Fe cations, and Fe-Fe bond polarization in BFCA. These observations provide direct support for the proposal that the large polarizability of the anions is critical to iron-based superconductivity by solvating the repulsive Coulomb interaction between electrons in the Fe plane [1].

Accurate determination of the doping and temperature dependences of the Fe-quadrupole and As-dipole polarizations requires a systematic study of valence electron distributions for different doping concentration and temperature. Usually the low-order SFs were measured by quantitative CBED, highorder SFs by single crystal x-ray diffraction (SCXRD). For FeSC, however, high quality single crystals are currently not available for SCXRD. We therefore use large angle CBED (LACBED, Fig. 2(a-c)) and precession electron diffraction (Fig. 2(e,f)) to measure high-order SFs.

\section{References:}

[1] C. Ma, L. Wu, W. Yin, H. Yang, H. Shi, Z. Wang, J. Li, C. C. Homes, and Y. Zhu, Phys. Rev. Lett. 112 (2014), p. 077001. 
[2] The work is supported by the U.S. DOE, under contract No. DE-SC0012704.

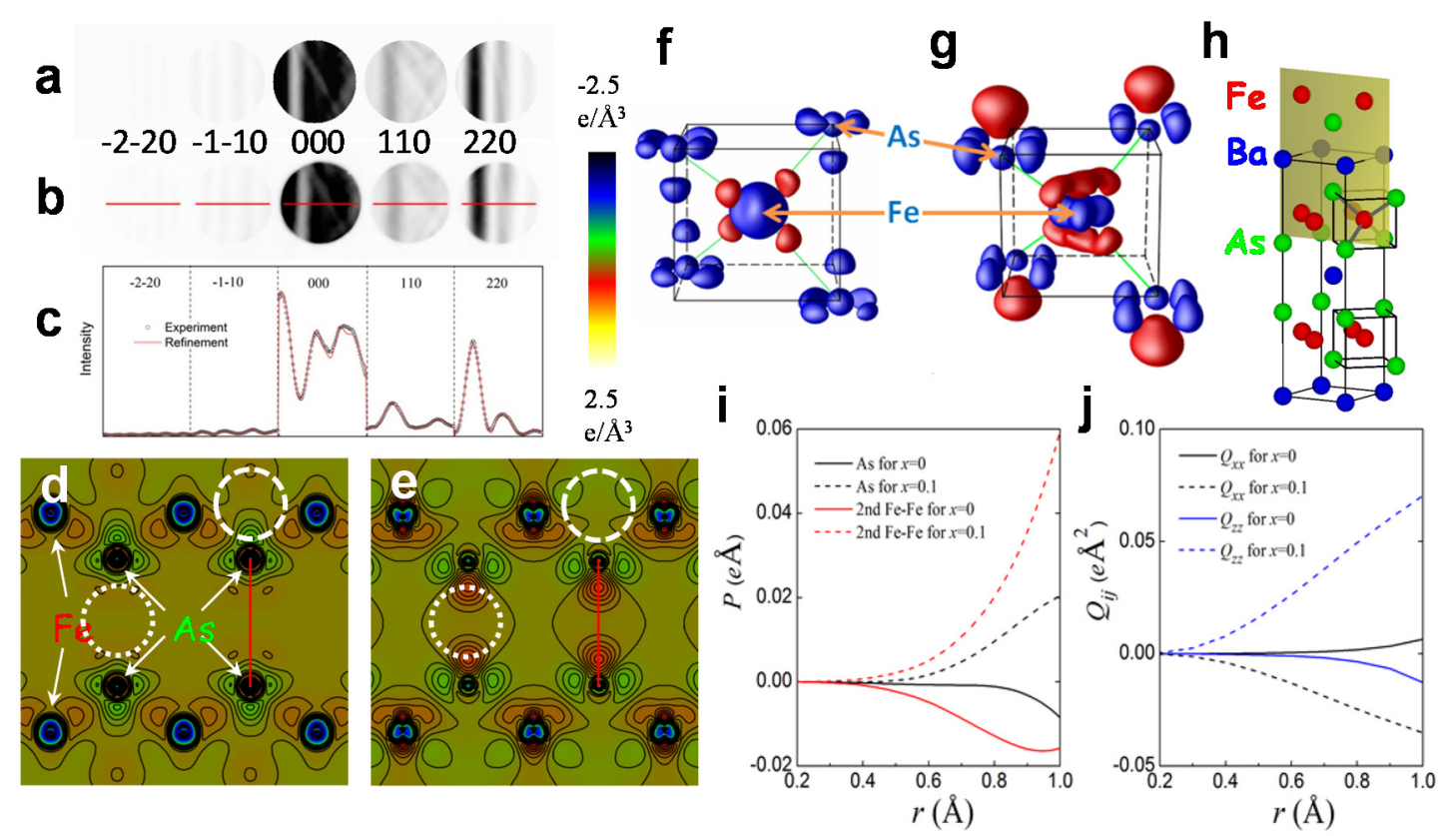

Figure 1. (a,b) Experimental energy-filtered (a) and calculated (b) CBED patterns of $\mathrm{BaFe}_{2} \mathrm{As}_{2}$ showing the $h h 0$ systematical row reflections at room temperature. (c) Line scans of the intensity profile from the experimental pattern (dots) and calculated one (solid line) after the structure factor refinement. (d,e) Valence electron density map of $\mathrm{Ba}\left(\mathrm{Fe}_{1-\mathrm{x}} \mathrm{Co}_{\mathrm{x}}\right)_{2} \mathrm{As} 2$ in (100) FeAs plane for (d) $\mathrm{x}=0$ and (e) $\mathrm{x}=0.1$ as well as the three-dimensional map for $(\mathrm{f}) \mathrm{x}=0$ and $(\mathrm{g}) \mathrm{x}=0.1$. The isovalues of difference charge density on the isosurface are $0.1 \mathrm{e} / \AA^{3}$ (red) and $-0.1 \mathrm{e} / \AA^{3}$ (blue), respectively. The color legend indicates the magnitude of the charge density. The red line denotes the shortest As-As distance between the FeAs layers. (h) The structure model. (i) The radius dependence of the out-of-plane dipole moment inside the sphere centered at the As anion and inside the sphere centered at the middle point of the next-nearest FeFe bond illustrated as the white dashed circle in (d) and (e) (red solid and dashed lines). (j) The radius dependence of the quadrupole moments inside the sphere centered at the $\mathrm{Fe}$ anion.

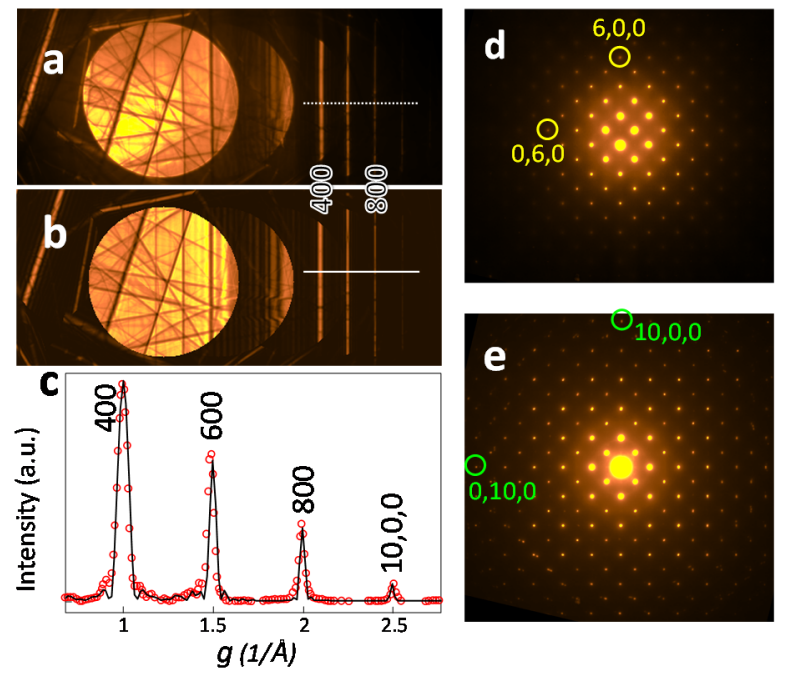

Figure 2. (a,b) Experimental energy-filtered (a) and calculated (b) LACBED patterns of $\mathrm{BaFe}_{2} \mathrm{As}_{2}$, showing the high-order reflections at $90 \mathrm{~K}$. (c) Intensity line profile from the experimental pattern (dots) and calculated one (solid line) after high-order structure factors refinement. (d,e) Electron diffraction patterns without precession (d) and with precession (e, precession angle $=3.1^{\circ}$ ) for $\mathrm{BaFe}_{2} \mathrm{As}_{2}$. With precession, not only more high-order reflections (up to 10,0,0) are measurable, but also their intensities are close to the structure factor squared, thus suitable for electron density refinement. 